\title{
Does Interleukin 22 Have a Role in Periodontal Pathogenesis? A Short Review
}

\author{
SreenivasNagarakanti ${ }^{1 *}$ and ArunKV ${ }^{2}$ \\ ${ }^{1}$ Department of Periodontology, Narayana Dental College \& Hospital, Nellore, INDIA \\ ${ }^{2}$ Department of Periodontology, Ragas Dental College \& Hospital, Chennai, INDIA.
}

\begin{abstract}
Many studies from recent years have shown that Interleukin 22 (IL-22) plays a major role in both the defence against certain microbes and the development and maintenance of chronic inflammatory diseases.Periodontal disease, including gingivitis and periodontitis, is caused by the interaction between pathogenic bacteria and the host immune system. Further animal and human studies are needed to investigate the role of IL-22 in periodontal health and disease.
\end{abstract}

Keywords: IL-22, Periodontitis, Host Defence, Inflammation

\section{Introduction}

Interleukin 22 (IL-22) is a member of the IL-10 family of cytokines (1). The cytokines of this family (IL-10, IL-19, IL-20, IL-24, IL-26, IL- 28 (a and b), and IL-29) display homologous secondary structures and 20-30\% amino acid identity. IL-22 was first described in 2000 as a gene differentially expressed in IL-9-treated BW5147 murine T lymphoma cells (2). IL-22 is mainly produced by activated T and NK cells. Highest IL-22 expression has been detected in CD4+ memory cells (3).

IL-22 acts on non-hematopoietic cells in epithelial tissues, which express a functional IL-22 receptor composed of an heterodimer of IL-22R1 and IL-10R2 $(4,5,6)$. IL-22 signalling triggers the production of antimicrobial peptides and pro-inflammatory molecules, and it also promotes tissue repair by inducing epithelial cell proliferation and survival (1-7). IL-22 has a role in the control of host defence against bacterial infections (8) and also limits tissue destruction in hepatitis (9), colitis and graft versus host disease (10) and drives endogenous thymic regeneration (11). Dysregulation of IL-22 signalling contributes to the pathogenesis of psoriasis (12), arthritis (13), and colon cancer (14).Generally, IL-22 acts to strengthen epithelial barrier functions and is involved both in tissue homeostasis as well as tissue repair and wound healing. However, uncontrolled excessive or prolonged production of IL-22 can cause pathology, such as psoriasis-like skin inflammation (15).

In general, IL-22 functions to sustain the integrity and barrier functions of many tissues and prevent damage caused by either invading pathogens or by the inflammatory response itself $(6,7)$. In this process, IL-22 can directly elicit proinflammatory epithelial defense mechanisms that are essential for host protection. However, if not tightly controlled, this bears the risk of IL-22 amplifying inflammation either alone or in synergy with other cytokines, and causing abnormal epithelial proliferation and differentiation, as seen in some infectious and inflammatory conditions (15).

\section{Functions of IL-22}

IL-22 promotes epithelial proliferation and helps to maintain and restore the integrity of the epithelial barrier function during the invasion of pathogens. It synergistically with other cytokines (IL-17/Tumor necrosis factor $\alpha$ ) induces the expression of antimicrobial proteins involved in host defense in the skin, the airways, and the intestine $(16,17)$. IL-22 also has a role in promoting the production of inflammatory mediators, such as IL-6, granulocyte colony-stimulating factor, IL-1 $\beta$, serum amyloid and lipopolysaccharide-binding protein (18-20). In addition to its antimicrobial and pro-inflammatory functions, IL22 contributes to tissue regeneration and promotes wound healing (21-23).

Due to lack of IL-22R expression on leukocytes, IL-22 does not induce anti-inflammatory response. Studies showed, forceful expression of IL-22 receptor on leukocytes results in pro-inflammatory symptoms in vivo (24). The anti-inflammatory activity of IL-22 is probably an indirect consequence of its tissue protective functions. In fact, IL-22 acts as a pro-inflammatory cytokine in many cases, and an uncontrolled expression of IL-22 can lead to pathology (15).

The double-headed role of IL-22 as pro and antiinflammatory function depends on many factors such as concentration and duration of local IL-22, the target tissues, and the cytokine environment $(16,25)$. 


\section{Discussion}

Many studies proved that the initiation of periodontal diseases is due to colonization of the gingival sulcus by pathogenic bacteria followed by a host response consisting of pro-inflammatory mediators and the infiltration of immune cells in an effort to rid the host tissue of the bacterial insult.

Research showed that IL-22 elicits various innate immune responses from epithelial cells and is essential for host defense against several invading pathogens and also protects tissue integrity and maintains the mucosal homeostasis. On the other handit acts as a proinflammatory cytokine that might result in tissue damage.

Till date there is no literature on the role of IL-22 in periodontal health and disease. However, further investigations are required to evaluate the effects IL-22 on periodontal tissues.

\section{References}

1. Ouyang W, Rutz S, CrellinNK, Valdez PA, HymowitzSG. Regulation and Functions of IL-10 Family Cytokines in Inflammation and Diseases. Annu Rev Immunol 2011;29:71-109.

2. Dumoutier, L., Louahed, J., and Renauld, J.C. (2000a). Cloning and characterization of IL-10-related T cell-derived inducible factor (IL- TIF), a novel cytokine structurally related to IL-10 and inducible by IL-9. J. Immunol. 164, 1814-1819.

3. Wolk, K., Kunz, S., Asadullah, K., and Sabat, R. (2002). Cutting edge: immune cells as sources and targets of the IL10 family members? J. Immunol. 168, 5397-5402.

4. Dumoutier L, Van Roost E, Colau D, RenauldJC. Human interleukin-10-related $\mathrm{T}$ cell-derived inducible factor: molecular cloning and functional characterization as an hepatocyte- stimulating factor. ProcNatlAcadSci USA 2000;97:10144-10149.

5. Xie MH, et al. Interleukin (IL)-22, a novel human cytokine that signals through the interferon receptor-related proteins CRF2-4 and IL-22R. J BiolChem 2000;275:31335-31339.

6. ZenewiczLA1, Flavell RA. Recent advances in IL22 biology. IntImmunol. 2011 Mar;23(3):159-63.

7. Sonnenberg, G. F., Fouser, L. A. \&Artis, D. Border patrol: regulation of immunity, inflammation and tissue homeostasis at barrier surfaces by IL-22. Nat. Immunol. 2011. 12, 383-390.

8. Aujla, S. J. et al. IL-22 mediates mucosal host defense against Gram-negative bacterial pneumonia. Nat. Med. 14, 275-281 (2008).
9. Zenewicz, LA. et al. Interleukin-22 but not interleukin-17 provides protection to hepatocytes during acute liver inflammation. Immunity 27,647-659 (2007).

10. Hanash, AM. et al. Interleukin-22 protects intestinal stem cells from immune- mediated tissue damage and regulates sensitivity to graft versus host disease. Immunity 37, 339-350 (2012).

11. Dudakov, J. A. et al. Interleukin-22 drives endogenous thymic regeneration in mice. Science 336, 91-95 (2012).

12. Zheng, Y. et al. Interleukin-22, a $\mathrm{T}(\mathrm{H}) 17$ cytokine, mediates IL-23-induced dermal inflammation and acanthosis. Nature 445, 648-651 (2007).

13. Sherlock, J. P. et al. IL-23 induces spondyloarthropathy by acting on ROR-gammat $\mathrm{p}$ CD3 $\mathrm{p}$ CD4-CD8- entheseal resident T cells. Nat. Med. 18, 1069-1076 (2012).

14. Huber, S. et al. IL-22BP is regulated by the inflammasome and modulates tumorigenesis in the intestine. Nature 491, 259-263 (2012)

15. Rutz S, Eidenschenk C, Ouyang W. IL-22, not simply a Th17 cytokine.Immunol Rev. 2013 Mar;252(1):116-32.

16. Liang SC, et al. Interleukin (IL)-22 and IL-17 arecoexpressed by Th17 cells and cooperatively enhance expression of antimicrobial peptides. J Exp Med 2006;203:2271-2279.

17. Wolk K, Kunz S, Witte E, Friedrich M, Asadullah K, Sabat R. IL-22 increases the innate immunity of tissues. Immunity 2004;21:241-254

18. Wolk K, et al. IL-22 regulates the expression of genes responsible for antimicrobial defense, cellular differentiation, and mobility in keratinocytes: a potential role in psoriasis. Eur J Immunol 2006;36:1309-1323.

19. Liang SC, et al. IL-22 Induces an Acute-Phase Response. J Immunol 2010;185:5531-5538.

20. Aujla S, et al. IL-22 mediates mucosal host defense against Gram-negative bacterial pneumonia. Nat Med 2008;14:275-281.

21. $\mathrm{Ki} \mathrm{S-H}$, et al. Interleukin-22 treatment ameliorates alcoholic liver injury in a murine model of chronic-binge ethanol feeding: role of signal transducer and activator of transcription 3. Hepatology 2010;52:1291-1300.

22. Kong $X$, et al. Interleukin-22 induces hepatic stellate cell senescence and restricts liver fibrosis in mice. Hepatology 2012;56:1150-1159.

23. Dudakov JA, et al. Interleukin-22 drives endogenous thymic regeneration in mice. Science 2012;336:91-95.

24. Savan R, et al. A novel role for IL-22R1 as a driver of inflammation. Blood 2011;117:575-584.

25. SonnenbergGF, Nair MG, KirnTJ, Zaph C, Fouser LA, Artis D. Pathological versus protective functions of IL-22 in airway inflammation are regulated by IL-17A. J Exp Med 2010;207:1293-1305.

*Corresponding author:

Dr. Sreenivas Nagarakanti, M.D.S, (PhD), Professor, Department of Periodontics, Narayana Dental College \& Hospital, ChinthareddyPalem, Nellore

AndhraPradesh, INDIA, Pin: 524003

Phone: +919985664566

Email: sreenivasnagarakanti@gmail.com

Date of Submission : 08.02.2017

Date of Acceptance : 19.02.2017

Financial or other Competing Interests: None.

Date of Publication : 21.02.2017 